\title{
The Thioredoxin Family Proteins: Histopathological Time Course Study in the Asphyctic Male Rat Brain
}

\author{
Aón Bertolino, Ma. Laura ${ }^{1-3^{*}}$, Christopher Horst Lillig' ${ }^{2}$ Capani Francisco ${ }^{3}$. \\ ${ }^{1}$ Federal Police Hospital, Pathology Department, C.A.B.A, Argentina; \\ ${ }^{2}$ Institute of Medical Biochemistry and Molecular Biology, University of Greifswald, Greifswald, \\ Germany. \\ ${ }^{3}$ Cardiological Research Institute "Prof. Dr. Alberto C. Taquini". UBA- CONICET. Laboratory of \\ Citoarquitectural and Neuronal Plasticity, C.A.B.A, Argentina. \\ *Corresponding author: lauraaon@hotmail.com.
}

Summary: Thioredoxin Family of proteins as Thioredoxin (Trxs), Glutaredoxins (Grxs) and Peroxiredoxins (Prxs) are one of the most important agents in the defense of oxidative stress and redox regulation. Perinatal asphyxia (AP) a disorder generated at the expense of the deficit of oxygen associated or not to ischemia, affects 5 to 10 of every 1,000 live births in developing country and is a serious health problem worldwide. Alterations in antioxidant protection systems are involved in the pathogenesis of hypoxic-ischemic insult and neuronal death. For these reasons it is proposed that the AP can cause changes in the distribution and expression of antioxidant proteins and enhance their deleterious or neuroprotective effects on the CNS. Methods: to determine the implication of the proteins role induced in the hypoxic brain injury, an animal model in vivo of PA was used in this work. In the first instance, the identification of the distribution of Trxs family proteins Trx1, Trx2, TrxR1, TrxR2, Txnip, Grx1, Grx2, Grx3, Grx5, $\gamma$-GCS, Prx1, Prx2, Prx3, Prx4, Prx5 and Prx6 was performed by immunohistochemistry on areas most sensitive to a hypoxia-ischemia insult: cerebellum, striatum, hippocampus, spinal cord, sustantia nigra, cortex and retina. Previous studies suggest that these proteins have an extensive and characteristic distribution in various cell types and regions of the CNS, although we observed significant differences in labeling intensity and distribution with conventional and fluorescence optical microscopy. After determining the cellular localization of Trxs, their behavior was studied by during a hypoxic- ischemic event by setting a time course at different times (2, 4, 6, 12, 24 and 72 hours post AP). Results: Trx1, Trx2, Grx1 and Grx2 proteins constitutes the main oxidoreductases in the cytosol and mitochondria, both in physiological and pathological conditions. Thus, for the short asphyxia times in which they were studied, Trx 1 was detected with increased expression at 2 hours and up to 4 hours post-AP, arguing a probable neuroprotective effect, although not enough, if taken into account tissue liability and high levels of free radicals detected at initial hours and the presence of structural damage at 7 days post-AP. Trx2 shows its increase at 6 and 12 hours and Grx1 at 24 hours. For Grx2, values elicited with the ELISA technique in the initial time curve were not representative in their expression in the hippocampus, contrary to the findings in the distribution in the striatum, where Grx2 was observed to be increased. Although at 7 hours post-AP levels begin to decline, these redoxins appears to be essential in the initial response to injury. Conclusions: by demonstrating of the presence of these enzymes in different cell types and specific regions of the brain in rats subjected to AP provided us with important morphological evidence base to study later some of the molecular mechanisms involved in the pathogenesis of cerebral ischemia that will help to generate new therapeutic tools to mitigate a disease whose neurological complications have no definitive solution. 

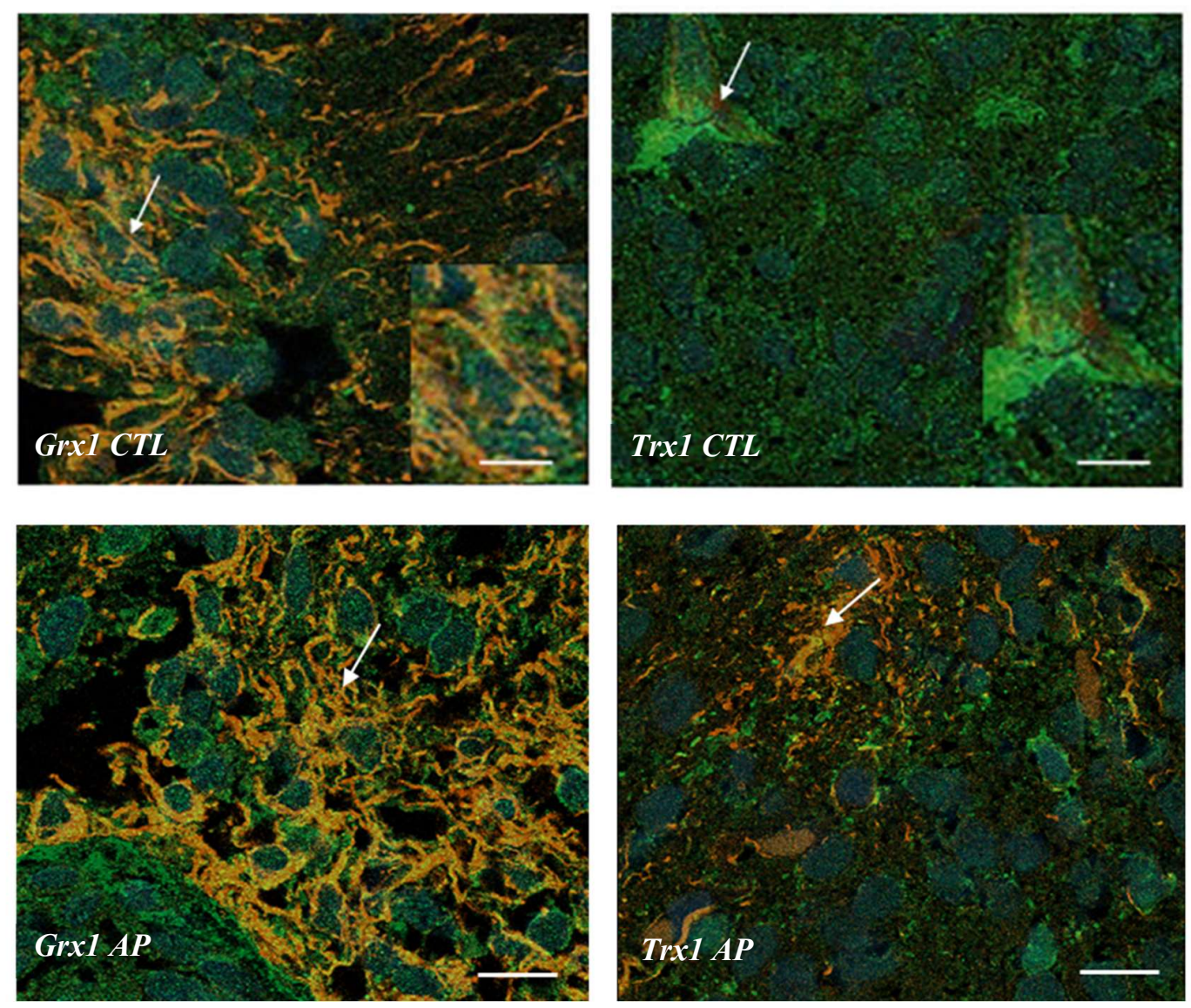

Figure 1: Direct immunofluorescence (IFD) techniques evaluated in confocal microscope with double staining of GFAP (acid gliofibrilar protein) and Thioredoxins under study were performed to determine associated reactive glial process in CTL and AP animals. Grx1 and Trx1 are shown here. (Scale: $20 \mu \mathrm{m}$. 400x).

\section{References}

[1] María Laura Aón Bertolino et al, Biochim Biophys Acta. 1810 (1). 93-100. (2011).

[2] Godoy J. R et al, Biochim Biophys Acta. 1810 (1). 2-92. (2011)

[3] Bjelke, B. et al, 1991. Brain Res. 543, 1-9.

[4] This Research was supported by CONICET Grant 11420100100159. And DAAD, in cooperation with Marburg University, Marburg, Germany.

[5] The authors acknowledge all the technical and discussion work of all the laboratory members.

[6] The authors declare no conflict of interest. 